Extended abstract submitted to:

AIAA Scitech 2019, San Diego, Ca

7-11 January 2019

Session: Applied Aerodynamics, Special Session: Space Launch System Aerosciences

\title{
Wind Tunnel Flow Field Visualizations of the Space Launch System Vehicle Ascent
}

\author{
Theodore J. Garbeff II* \\ NASA Ames Research Center, Moffett Field, Ca, 94035
}

\begin{abstract}
A wind tunnel test was conducted to characterize the aeroacoustic environment of several configurations of the Space Launch System during ascent. The test was conducted in the 11-by-11 foot transonic and 9-by-7 foot supersonic test sections at NASA Ames research center. Throughout this experiment data was collected from several types of instrumentation including: dynamic and steady-state pressure sensors, unsteady and steady pressure sensitive paint, time-resolved shadowgraph and infrared imaging. The following details results and analysis from the time-resolved shadowgraph and infrared imaging data systems. The time-resolved shadowgraph provided a qualitative measurement of the near-field turbulent fluctuations. These results helped provide context to the relative magnitude and frequency content of the fluid-structure-interaction driving the surface pressure phenomena characterized by the discrete pressure transducers and unsteady pressure sensitive paint. The infrared imaging was used to verify boundary layer trip effectiveness and provide temperature correction for the unsteady pressure sensitive paint.
\end{abstract}

\begin{abstract}
A I. Introduction

1 wind tunnel test was conducted to characterize the aeroacoustic environment of several configurations of the Space Launch System (SLS) during ascent. The test was conducted in the 11-by-11 foot transonic and 9-by-7 foot supersonic test sections of the Unitary Plan Wind Tunnel (UPWT) at NASA Ames research center. Throughout this experiment data was collected from several types of instrumentation including: dynamic and steady-state pressure sensors, unsteady and steady pressure sensitive paint, time-resolved shadowgraph and infrared imaging. The shadowgraph imaging systems at the NASA Ames UPWT consist of both high-speed and low-speed cameras that simultaneously image the flow field. This dual camera configuration produces two data products: A high-speed burst of images, and a high-resolution, long duration movie. The high-speed image burst is typically run at frame rates on the order of 50,000 with exposure times as low as 4 microseconds. This enables the ability to time-resolve flow features and perform statistical and frequency domain analysis on the flow-field surrounding the vehicle. The low-speed, high-resolution movie captures the content that occurs between set-points as the model moves in in angle-ofattack or sideslip. This is useful for review purposes and can help verify test repeatability or identify low-frequency flow phenomena such as hysteresis. Shadowgraph imagery was captured on every model configuration in each test section at Mach numbers ranging from 0.7 to 2.5. In addition to the dual shadowgraph data systems the Ames UPWT possesses an advanced infrared flow visualization capability. Consisting of high sensitivity, megapixel mid-wave infrared cameras and unique contrast enhancement algorithms, this system is capable of providing real-time imaging of surface flow features. These flow features include boundary layer transition, shock impingement, flow separation and buffet. Infrared imagery was captured during a subset of the wind tunnel test corresponding with the unsteady pressure sensitive paint measurements. The primary goal of these measurements was to record the variation in temperature across the wind tunnel model, providing a dataset to temperature correct the unsteady pressure sensitive paint. Any other flow features visualized using the infrared technique were secondary objectives.

Figure 1 details a shadowgram of the $2.5 \%$ configuration of the SLS wind tunnel model at transonic conditions in the 11by-11 foot test section. As the flow accelerates around the inter-stage adapter a strong shock forms (left portion of image). Flow disturbances due to the flanges on the center body disrupt the boundary layer and create regions of elevated fluctuations in the near-field. In addition to the flow features that are created by the presence of the model, there are phenomena visible in Figure 1 that are produced by the operation of a transonic wind tunnel. These features are produced by acoustic phenomena driven by the slotted test section walls (slot tones) and blade pass frequency of the three-stage compressor (drive tones) occurring at different scales in space and time. This creates an information rich dataset that can often be overwhelming to the observer.
\end{abstract}

* Aerospace Engineer, Wind Tunnel Systems Branch, MS 227, Member AIAA

1

American Institute of Aeronautics and Astronautics 


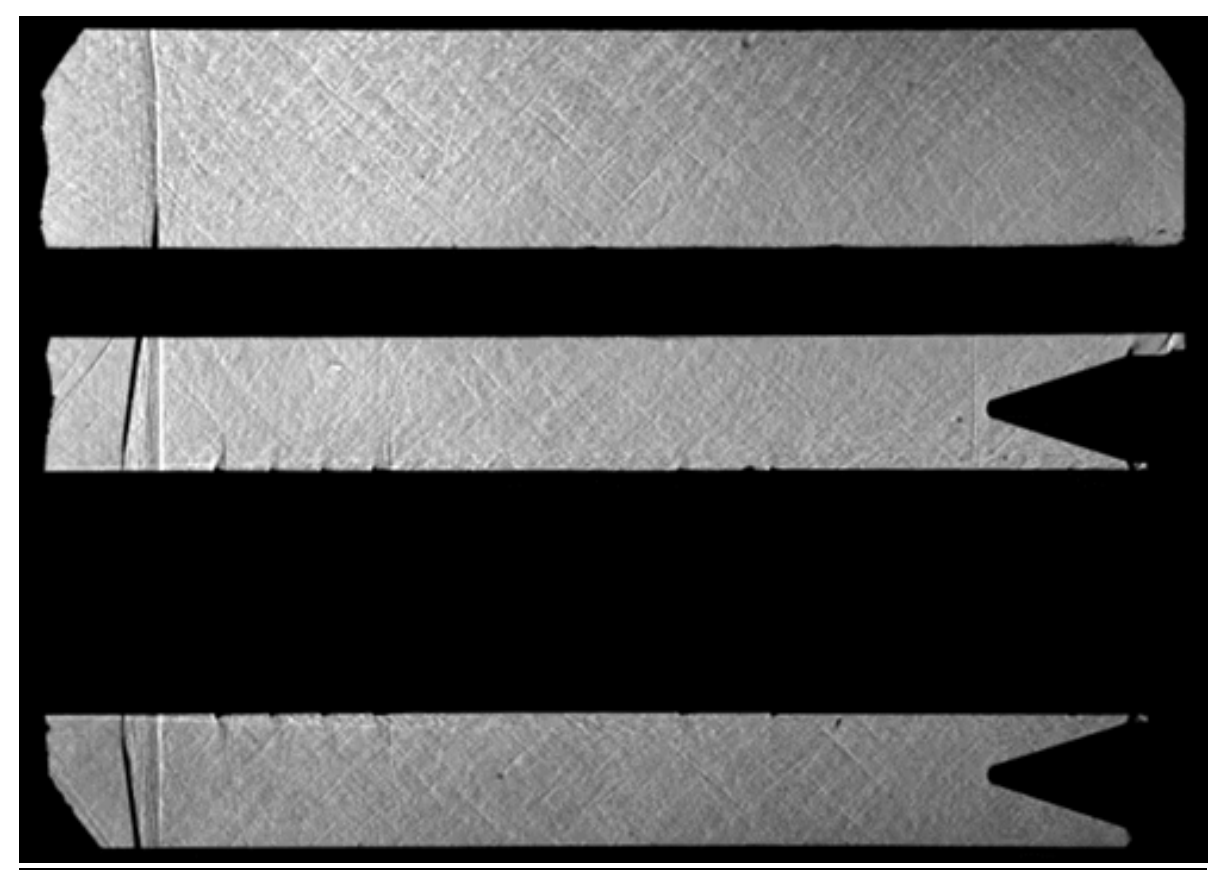

Figure 1: Shadowgram of the $2.5 \%$ configuration in 11-by-11 foot test section

The following outlines a technique to use frequency domain analysis and filtering to analyze these datasets. Often this results in a simplified representation of the dataset but can also reveal hidden features in the flow, adding insight and context regarding the underlying mechanisms for the researcher. The technique utilized the high-speed shadowgraph data system and is based on traditional frequency domain analysis where each pixel can be thought of as a sensor producing a time-series of data (pixel intensity). The following data was collected at 54,000 frames per second with 4 micro-second exposure times. A Fast Fourier Transform (FFT) is computed at each pixel location. The energy at each pixel location for a fixed frequency is summed across all pixels in the image resulting in a frequency map (Figure 2) of the total energy in the image. Figure 2 gives insight into the time scales that dominate the flow field. Based on the distribution of the image energy the frequency content can be split into three unique bands. A low-frequency band containing large amounts of energy, a mid-frequency band consisting of an energy plateau, followed by a high-frequency band characterized by several well-defined peaks. Having created a frequency map and used it to identify bands of interesting content, we can now apply band-pass filters to the original image data.

In a similar fashion to the aforementioned frequency domain analysis each pixel is treated as a sensor with time-series of data (pixel intensity). Low, mid, and high frequency band-pass filters are applied based on a frequency range identified by the frequency map shown in Figure 2. The result is three filtered image data sets, each representing a unique frequency band. This method results in a simplified data product where the features in the image data correspond with the time scale producing that feature. For example, Figure 3 details a comparison between unfiltered and filtered image data for the low frequency band. Here it is clear that the strong shock features are major contributors to the energy occurring in this frequency band. Conversely, the highfrequency filtered data identifies the features in the flow field contributing to that band. This can be seen in Figure 4 where the filtered image is now dominated by the many transverse waves associated with the slot tones of the test section wall. To reiterate each of these filtered images are derived from the same original dataset, this process has only separated what flow features are happening at different timescales.

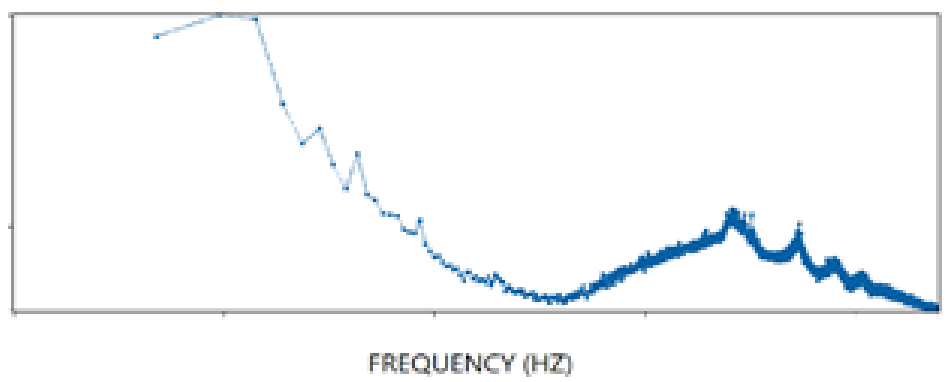

Figure 2: Image energy frequency map 

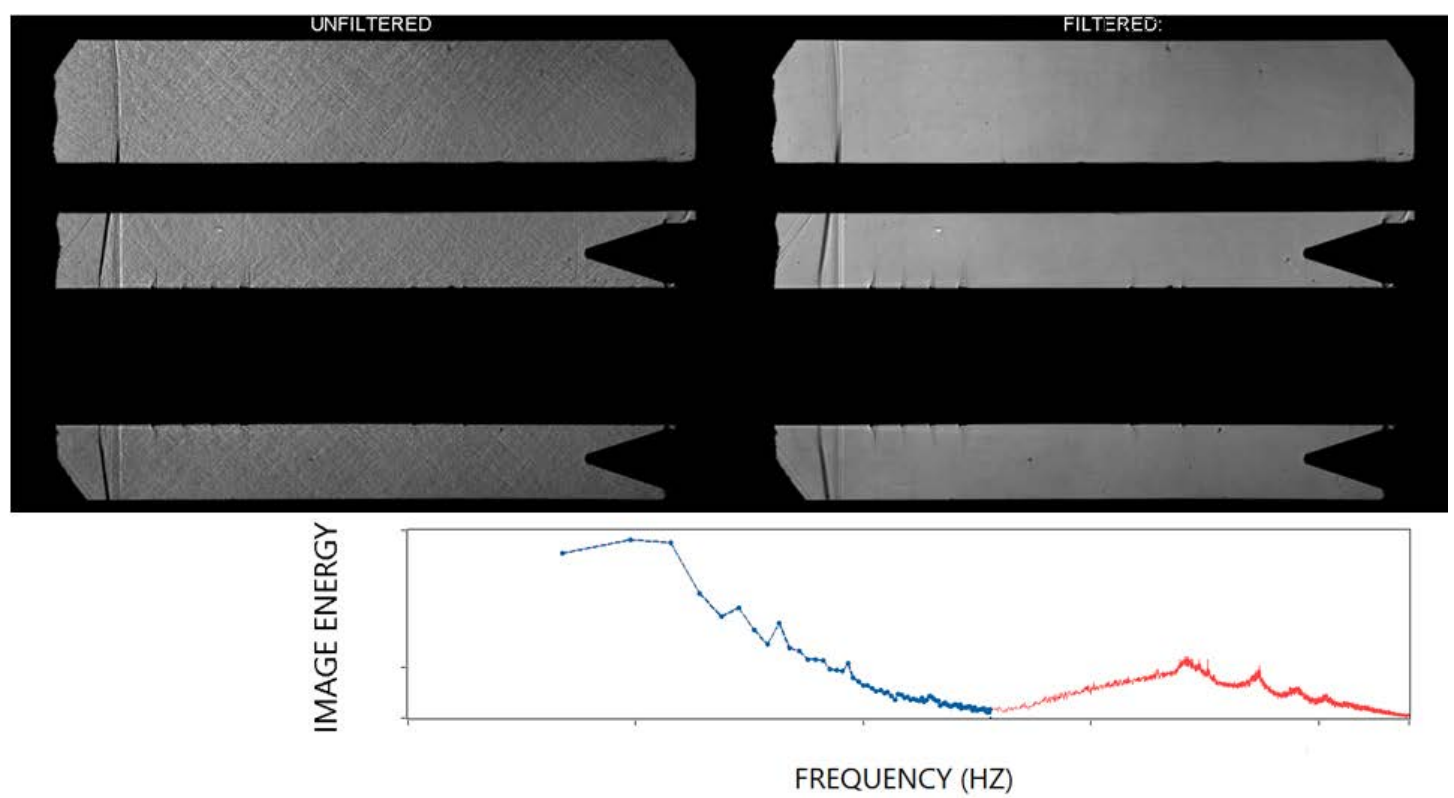

Figure 3: Filtered (blue region) image comparison (Low-frequency)

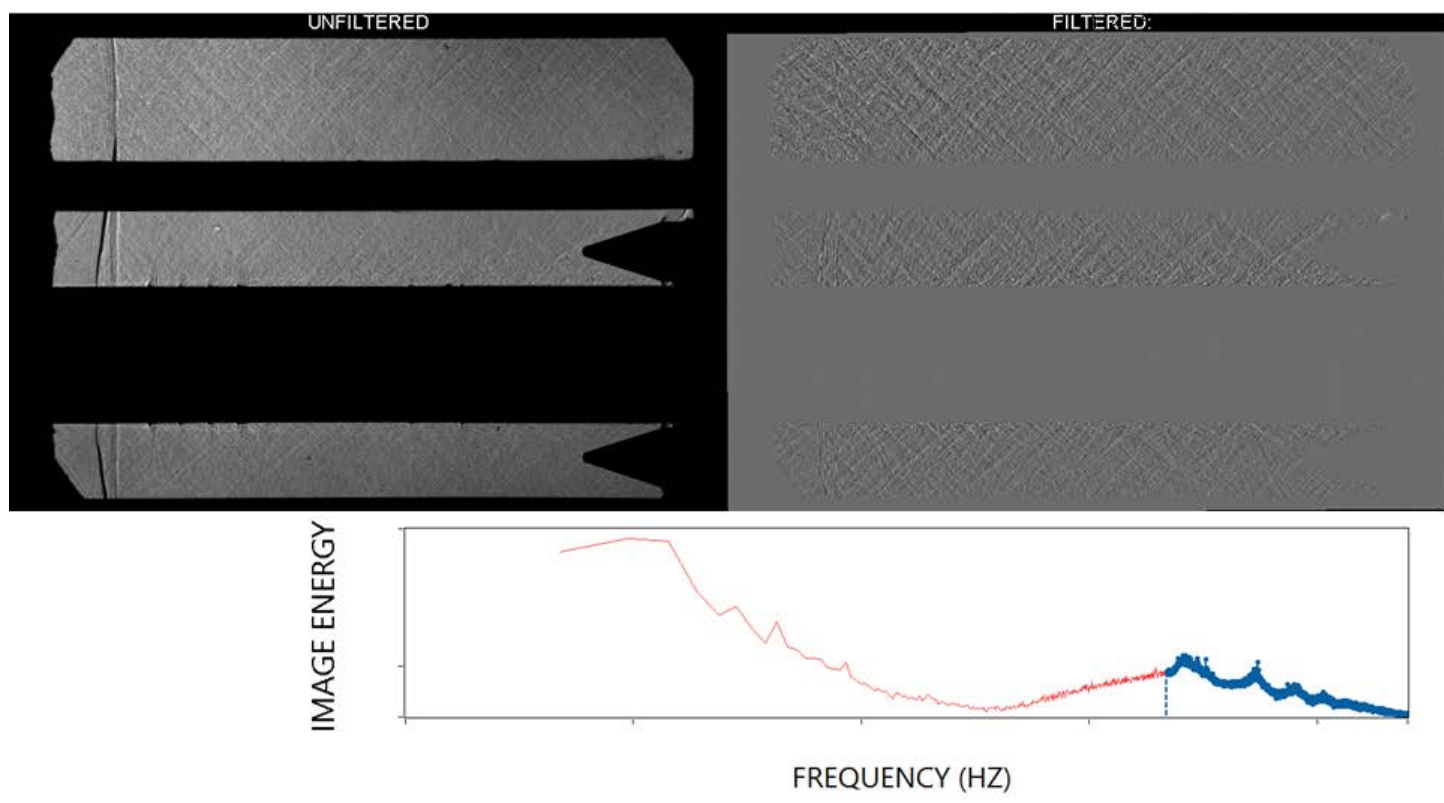

Figure 4: Filtered (blue region) image comparison (high-frequency)

Filtering the high-speed shadowgraph data also creates the ability to reveal features in the flow field that may otherwise be hidden. For example, given a drive speed the blade pass frequency 11-by-11 foot three stage compressor can be estimated. This blade pass frequency produces a measureable effect on the flow field. These drive tones have been well documented in both test sections using hot-wire anemometry and unsteady pressure transducers. In an attempt to visualize this drive tone frequency, a band-pass filter can be applied to the image data in the same fashion as previously discussed. Figure 5 details the results of filtering the image data in this frequency range. In this filtered image the drive tones are manifested as vertical waves that propagate upstream. This demonstrates the power of this technique to reveal structure within the flow that would otherwise be hidden. 

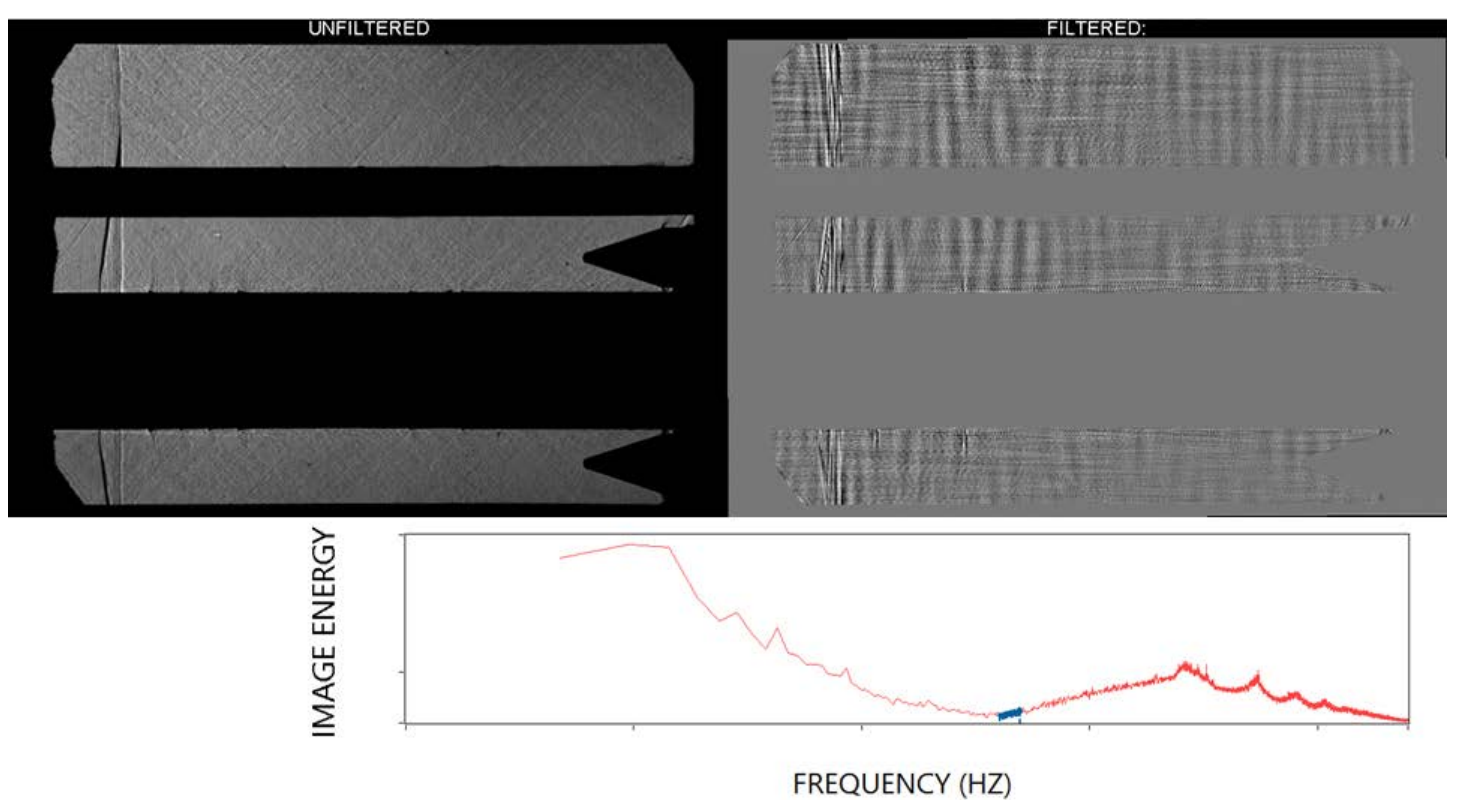

Figure 5: Filtered (blue region) image comparison (blade pass frequency)

Figures 3 and 4 compare the effects of filtering on a single image frame. This provides a quick comparison of the important features at that instance in time but does not reflect the effects of filtering on those structures that change with time. In order to capture and compare the effects of filtering on unsteady phenomena, the standard deviation is computed at each pixel location across the entire time series. Colorized contours of the low, mid, and high frequency filtered image pixel time history standard deviation are presented in Figures 6, 7, and 8. The low frequency standard deviation contour plot demonstrates how the strong shock features dominate the flow field across time. In the mid-frequency band the strong shock features are blurred and the weaker shock structures around the flanges of the center core are evident. This indicates that in this frequency range much of the energy is radiating from the shock structures. Additionally the growth of the boundary layer along the vehicle can now be seen. The high-frequency band color map shows elevated levels of pixel variation in the region distant from the vehicle. This indicates the prevalence of the slot tones on the image data. Also, the broadband nature of the boundary layer and turbulence created within shock structure is demonstrated as these regions contain energy out into higher frequencies.

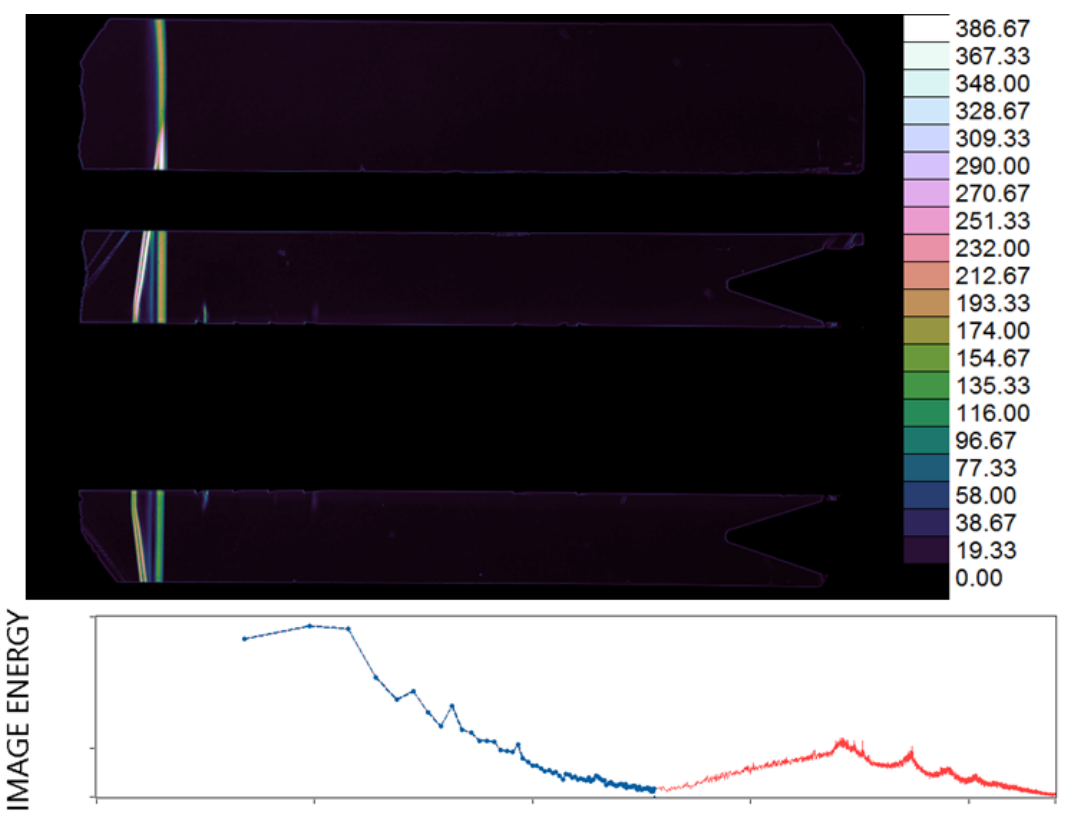

FREQUENCY $(\mathrm{HZ})$

Figure 6: Filtered pixel time history standard deviation (low-frequency) 

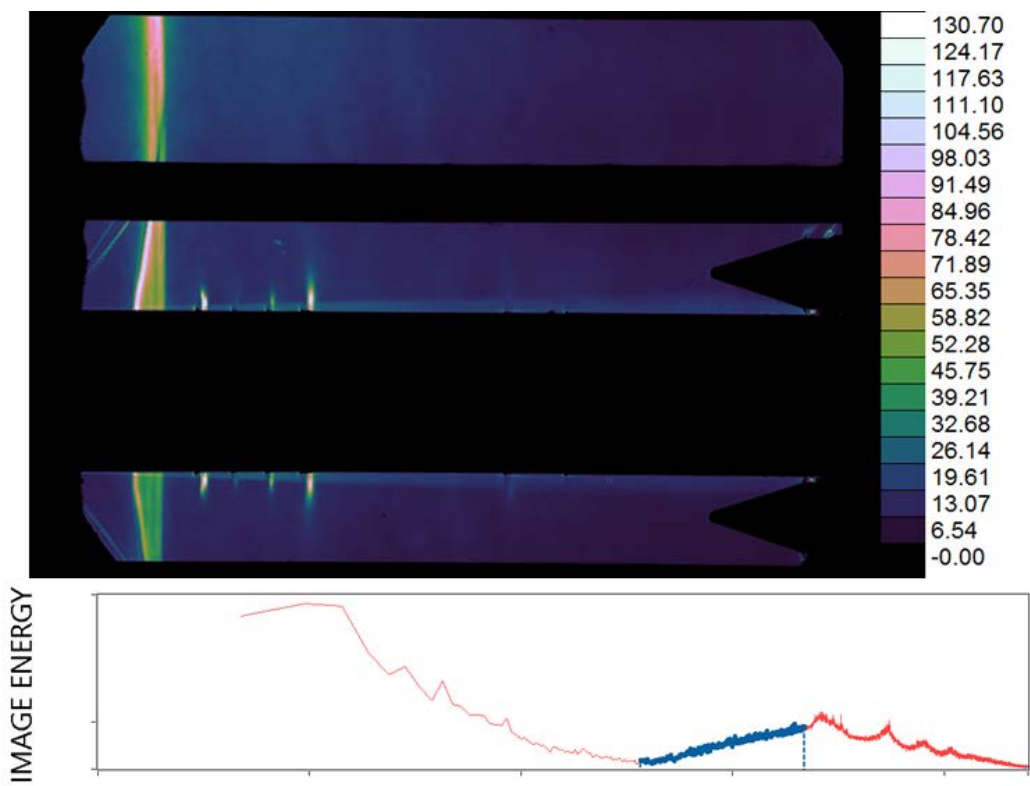

FREQUENCY (HZ)

Figure 7: Filtered pixel time history standard deviation (mid-frequency band)

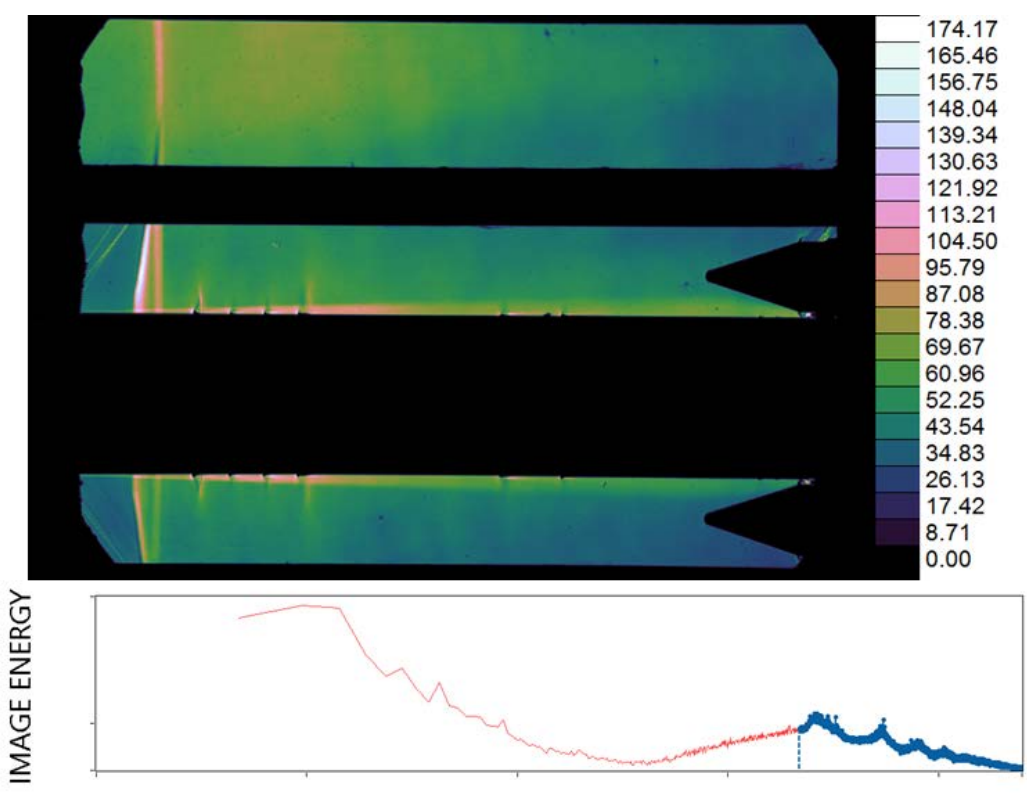

FREQUENCY (HZ)

Figure 8: Filtered pixel time history standard deviation (high-frequency band)

Infrared flow visualization relies on resolving small temperature gradients on the surface of wind tunnels models that are imparted by some aerodynamic phenomenon. The ability to image these small temperature gradients is highly dependent on the heat transfer conditions between the free stream and test article as well as the emissivity of the wind tunnel model. For a wind tunnel model with poor emissivity or non-ideal heat transfer conditions, this leads to the patterns of interest being hidden within a larger signal. This makes infrared flow visualization an excellent candidate for the preceding frequency domain analysis. Therefore the frequency domain analysis and filtering tool was adapted to process high-bit depth infrared images, resulting in some unexpected results. Figure 9 details two frequency images of the frequency map computed from the infrared time-series. The content in each image is separated by only a few hertz however the image on the left shows a shock structure around the vehicle while the image on the right does not. Applying a band pass filter to the original time series to select only the frequency content containing the shock features results in Figure 10. In this image the shock structure surrounding the vehicle is clearly evident yet is not visible in the original infrared image data. 

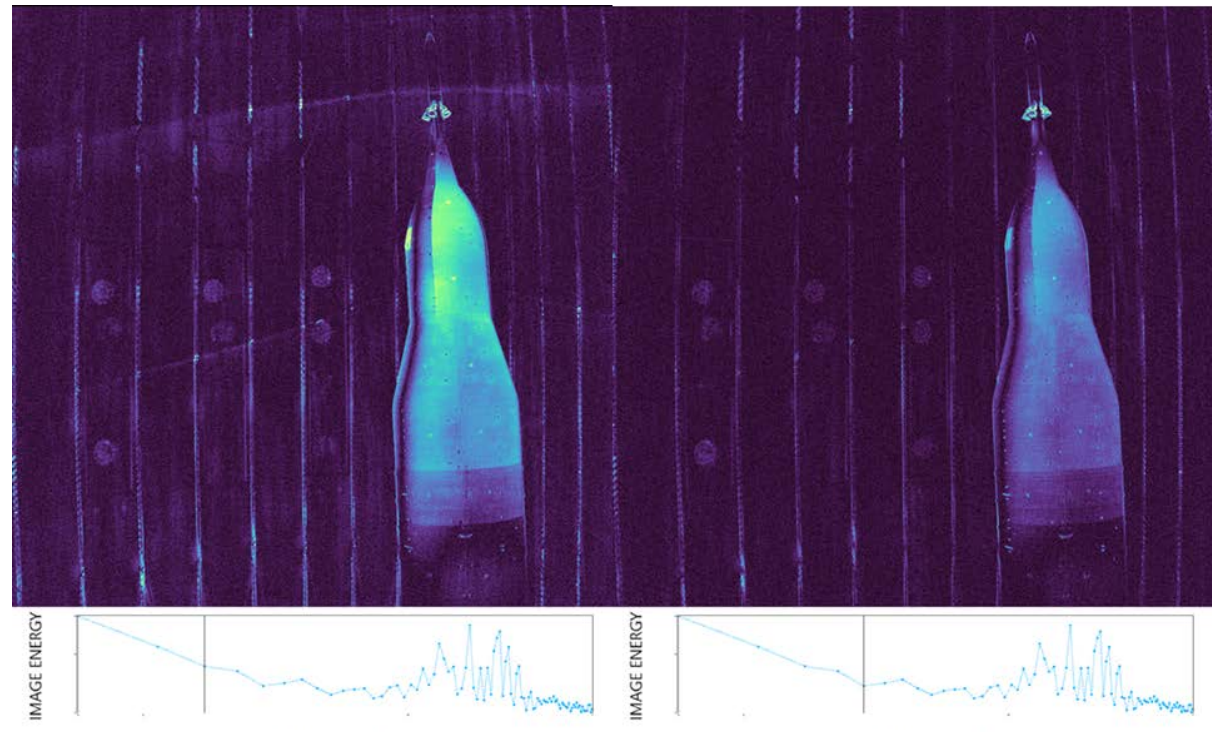

FREQUENCY (HZ)

FREQUENCY (HZ)

Figure 9: Infrared frequency map (two frequency instances)

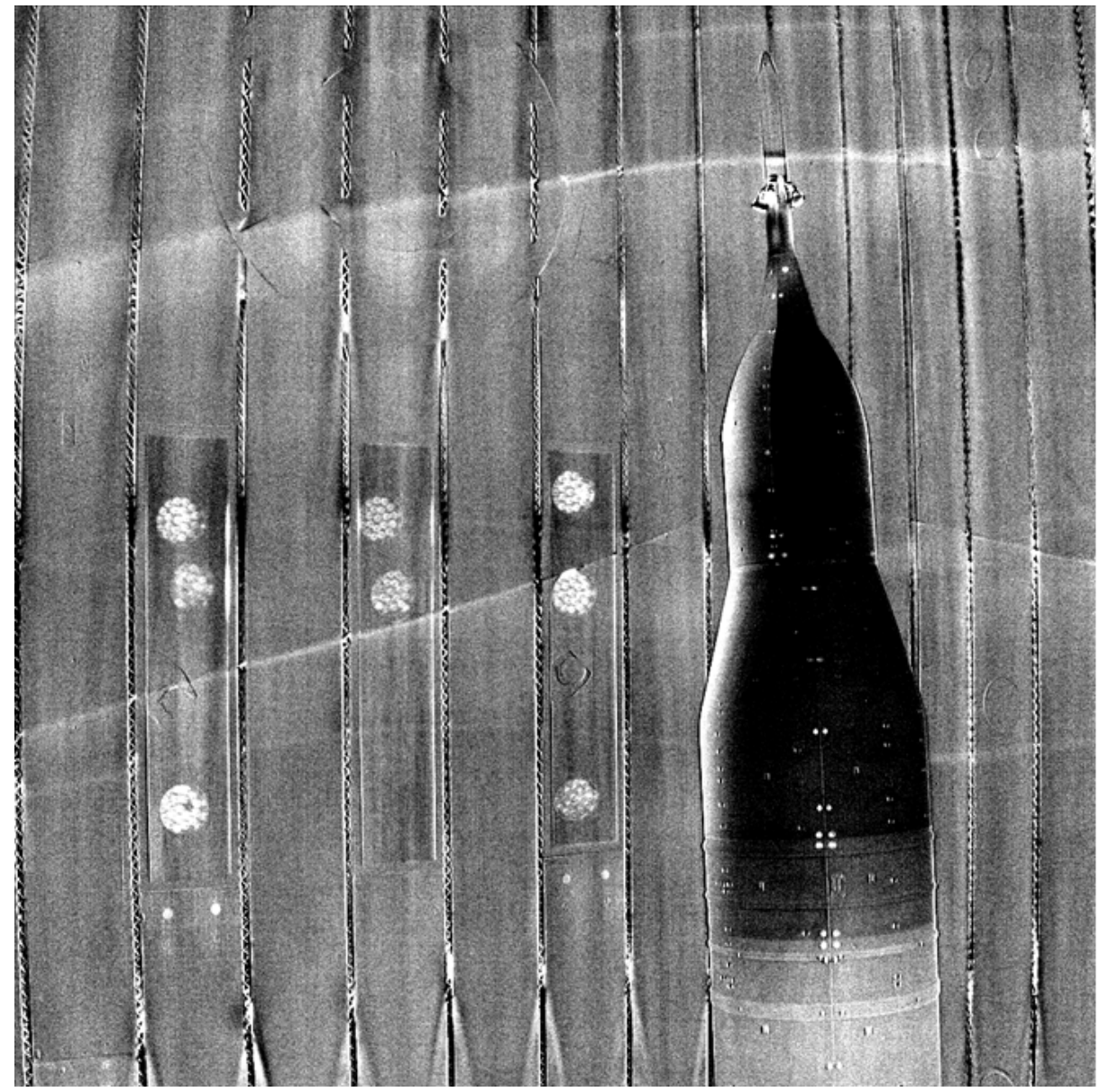

Figure 10: Band-pass filtered infrared image, reveals hidden shock structure 